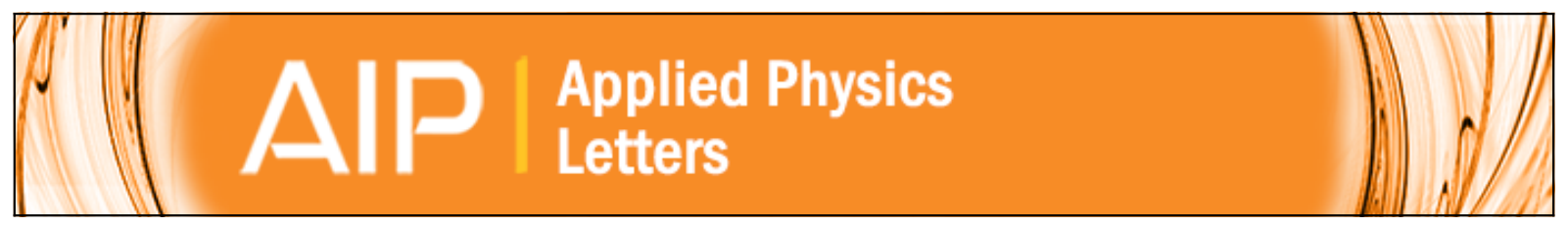

Spin polarization and barrier-oxidation effects at the Co alumina interface in magnetic tunnel junctions

N. D. Telling, G. van der Laan, S. Ladak, and R. J. Hicken

Citation: Applied Physics Letters 85, 3803 (2004); doi: 10.1063/1.1812383

View online: http://dx.doi.org/10.1063/1.1812383

View Table of Contents: http://scitation.aip.org/content/aip/journal/apl/85/17?ver=pdfcov

Published by the AIP Publishing 


\title{
Spin polarization and barrier-oxidation effects at the Co/alumina interface in magnetic tunnel junctions
}

\author{
N. D. Telling ${ }^{\text {a) }}$ and G. van der Laan \\ Magnetic Spectroscopy Group, CCLRC Daresbury Laboratory, Warrington WA4 4AD, United Kingdom \\ S. Ladak and R. J. Hicken \\ School of Physics, University of Exeter, Stocker Road, Exeter EX4 4QL, United Kingdom
}

(Received 21 July 2004; accepted 1 September 2004)

\begin{abstract}
The electronic structure and polarization in magnetic tunnel junctions prepared with varying degrees of barrier-layer oxidation have been studied using x-ray absorption spectroscopy across the Co $L_{2,3}$ absorption edges. It was found that the Co electronic structure near the Co/alumina interface tended to that of cobalt oxide as the barrier oxidation time was increased. However, the net Co $3 d$ spin polarization, determined from x-ray magnetic circular dichroism, increased for moderate oxidation times compared to that obtained for an under-oxidized $\mathrm{Co} / \mathrm{Al}$ interface. It is proposed that the expected dilution of the measured polarization due to the formation of (room temperature) paramagnetic cobalt oxide, is offset by an increase in the Co $3 d$ spin-polarization of the interface layer as the interface bonding changes from $\mathrm{Co}-\mathrm{Al}$ to $\mathrm{Co}-\mathrm{O}$ with increasing oxidation times. () 2004 American Institute of Physics. [DOI: 10.1063/1.1812383]
\end{abstract}

Since the first observation of large $(>10 \%)$ roomtemperature (RT) tunneling magnetoresistance (TMR) effects in magnetic tunnel junctions (MTJs), ${ }^{1}$ there has been considerable interest in understanding the factors that control the degree of tunneling spin-polarization (TSP) in these structures, with particular regard to potential applications in the areas of spintronics and magnetic recording. Although it is now well established that the sign and magnitude of the TSP does not depend only on the bulk spin polarization of the ferromagnet, the relative influence of contributing factors such as evanescent barrier states, interface bonding, and insulator disorder, is still a matter for rigorous investigation. ${ }^{2}$ In particular, in order to explain the positive TSP measured in Co/Alumina based $\mathrm{MTJs}^{3}{ }^{3}$ many theories point to the importance of the spin-polarized Co/alumina interface states and their different decay lengths within the barrier. ${ }^{4,5}$ Firstprinciples calculations have indicated that the formation of $\mathrm{Co}-\mathrm{O}$ interfacial bonds leads to an induced $\mathrm{O}$ interface moment of $0.07 \mu_{B}$ /atom without quenching the Co interface polarization. ${ }^{6}$ This is a result of the bonding between the exchange split $d$ orbitals of Co and $p$ orbitals of O. However, no such interfacial moment on barrier-layer atoms (either $\mathrm{O}$ or $\mathrm{Al}$ ) was predicted when $\mathrm{Co}-\mathrm{Al}$ interfacial bonds were considered. In this case the Co interface moments were reduced below the bulk value due to charge transfer from the interfacial $\mathrm{Al}$ atoms to the Co minority $3 d$ band.

The above-mentioned calculations indicate that the formation of $\mathrm{Co}-\mathrm{O}$ interface bonds is crucial for mediating spin-polarized tunneling in Co/alumina based junctions, via spin-polarized interface states. The nature of the interface bonding will be influenced by the preparation conditions for the alumina barrier, which have a pronounced effect on the TMR. ${ }^{7,8}$ For under-oxidized barriers (short oxidation times), in which there is a prevalence of $\mathrm{Co}-\mathrm{Al}$ interface bonds, the TMR is found to be poor. However, lengthy barrier oxidation times also lead to low TMR values despite an expected abun-

${ }^{a) E l e c t r o n i c ~ m a i l: ~ n . d . t e l l i n g @ d l . a c . u k ~}$ dance of $\mathrm{Co}-\mathrm{O}$ interface bonds. ${ }^{7,8}$ In this case it is thought that the formation of RT paramagnetic $\mathrm{CoO}$ or $\mathrm{Co}_{3} \mathrm{O}_{4}$ at the interface leads to the loss of electron spin polarization in the ferromagnet. In fact several recent studies using $\mathrm{x}$-ray absorption spectroscopy (XAS) have confirmed the formation of cobalt oxide in cobalt layers covered by thin alumina layers. ${ }^{9,10}$ In this letter we investigate the role of barrier oxidation time on interface polarization and TMR in MTJs, utilizing XAS techniques to explore both the electronic and magnetic structure of $\mathrm{Co}$ atoms near the Co/alumina interface.

MTJs were prepared using a combination of magnetron sputtering and plasma oxidation, described in detail elsewhere. ${ }^{11}$ Two nominally identical junctions were formed in each sample on polished quartz substrates with the layer sequence $\mathrm{SiO}_{2} / \mathrm{Ni}_{81} \mathrm{Fe}_{19}(80 \AA) / \mathrm{Co}(20 \AA) / \mathrm{Al}(20 \AA$ +plasma oxidation for $X$ seconds $) / \mathrm{Ni}_{18} \mathrm{Fe}_{19}(100 \AA) / \mathrm{Cu}(30 \AA)$. A series of MTJs was obtained with $X$ varied as $0,100,300$, and $600 \mathrm{~s}$. For the $0 \mathrm{~s}$ sample, only ex situ natural oxidation of the barrier in air could occur and this sample can thus be considered as the limiting under-oxidized junction.

XAS measurements were performed across the Co $L_{2,3}$ absorption edges using $85 \%$ circularly polarized $\mathrm{x}$ rays on beamline 1.1 at the SRS, Daresbury Laboratory. The x-ray beam was incident on the lower $\mathrm{NiFe} / \mathrm{Co}$ electrode covered by the alumina barrier, as shown schematically in the inset of Fig. 1. Absorption spectra were obtained in total-electronyield mode by measuring the drain current via the lower electrode contact pads. This method enhances the sensitivity to the Co/alumina interface owing to the limited electronescape depth. Additionally, by limiting the thickness of the Co layer directly beneath the alumina to $20 \AA$, the interface region comprises a greater proportion of the measured volume. The $x$ rays were incident at an angle of $45^{\circ}$ with respect to the sample plane and the $\mathrm{x}$-ray magnetic circular dichroism $(\mathrm{XMCD})^{12}$ was recorded as the difference in absorption upon reversing the film saturation magnetization in an external field of $\pm 0.6 \mathrm{~T}$ parallel to the incident beam. This field provides an in-plane component far exceeding the saturation 


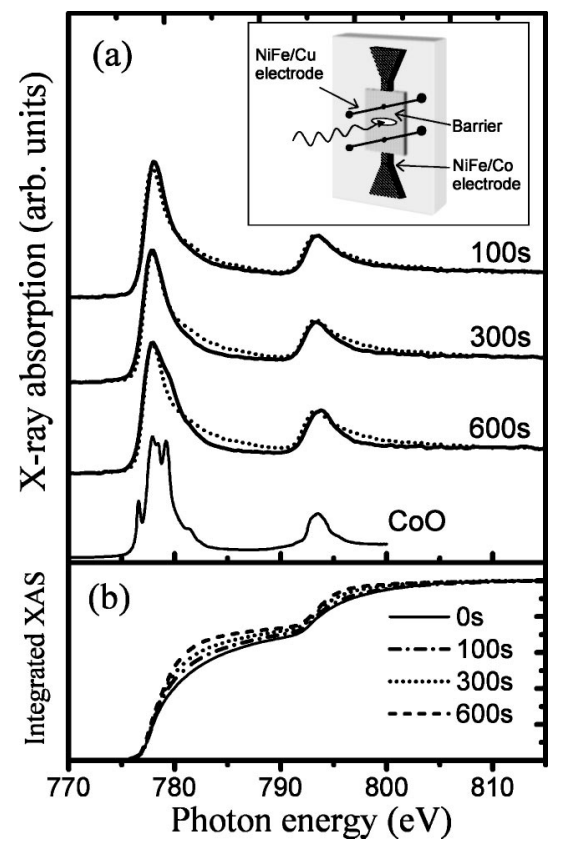

FIG. 1. (a) Co $L_{2,3} \mathrm{XAS}$ as a function of barrier oxidation time (solid lines), and compared to a $\mathrm{Co} / \mathrm{Al}$ interface ( $0 \mathrm{~s}$ oxidation time-dotted curve) and a $\mathrm{CoO}$ standard (Ref. 13). (b) Integrated XAS intensity (normalized to unity above the $L_{2}$ absorption edge) as a function of oxidation time. The inset shows schematically the MTJ configuration and geometry for XAS measurements.

field for these samples $(\lesssim 10 \mathrm{mT})$. Current-voltage $(I-V)$ and TMR measurements were obtained from the junctions using a conventional dc four-point probe technique.

The XAS spectra, obtained by averaging the curves for the two magnetization configurations, are shown for different oxidation times in Fig. 1(a). By comparing these spectra with those from the under-oxidized $\mathrm{Co} / \mathrm{Al}$ interface (dotted curve) and a $\mathrm{CoO}$ standard, ${ }^{13}$ a gradual change from metallic cobalt to cobalt oxide is evident. In particular, two main features are apparent with increasing oxidation time: (i) a gradual change in the line shape of the curve toward that of $\mathrm{CoO}$ (most noticeable by the decrease in intensity between the $L_{3}$ and $L_{2}$ absorption edges), (ii) the appearance of a broad shoulder at $\sim 780 \mathrm{eV}$, just above the main $L_{3}$ resonance peak. This latter feature is likely to be the emergence of the cobalt oxide multiplet structure, as seen by comparison with the curve from the $\mathrm{CoO}$ standard.

Further evidence of a change from cobalt metallic to oxide character can be found by examining the integrated XAS intensities, as shown in Fig. 1(b). The variation in the shape of these curves reflects the changes in line shape seen in Fig. 1(a). The branching ratio, $B$, is defined as $I\left(L_{3}\right) /\left[I\left(L_{2}\right)+I\left(L_{3}\right)\right]$, where $I$ is the integrated intensity. Although it is not obvious exactly where to take the end point of the $L_{3}$ absorption edge and the start of the $L_{2}$ edge in these curves, wherever we set this point in Fig. 1(b), $B$ can be seen to increase gradually with oxidation time. This is a consequence of the larger $3 d$ spin-orbit interaction in the cobalt oxide compared to the metal, ${ }^{14}$ yielding higher values of $B$.

In order to probe the magnetic structure of the Co atoms near the Co/alumina interface, we examined the XMCD in the absorption spectra. The XAS and corresponding XMCD curves obtained from the 0 and $600 \mathrm{~s}$ junction samples are shown in Figs. 2(a) and 2(b), respectively. The XAS curves were normaiized to the incident flux and scaled to a unit edge

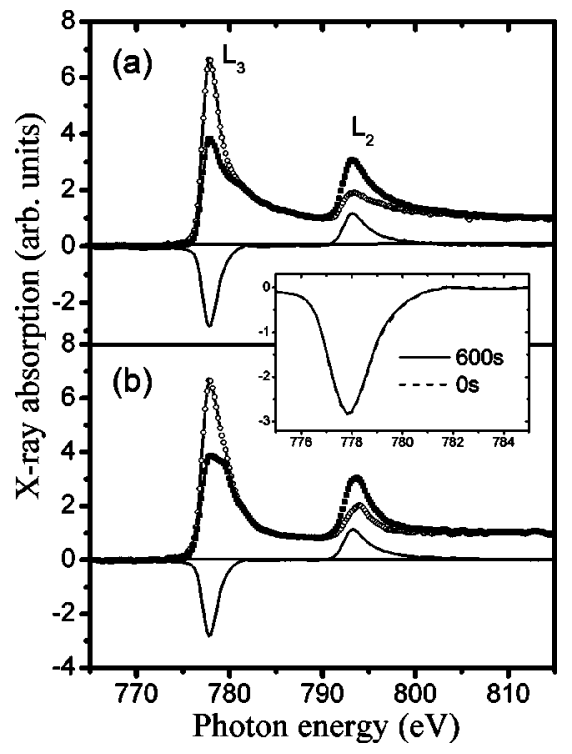

FIG. 2. Co $L_{2,3}$ XAS measured for the two saturation magnetization directions (symbols) and the resulting XMCD spectra (solid lines) for (a) the $\mathrm{Co} / \mathrm{Al}$ interface ( $0 \mathrm{~s}$ oxidation time) and (b) the $600 \mathrm{~s}$ oxidation time sample. The inset shows an enlargement of the XMCD around the $L_{3}$ edge.

jump. The intensities shown are corrected for the degree of polarization and the angle between the magnetization direction and incident beam. In addition, a linear background was removed.

It is apparent that, despite significant differences between the XAS curves in Fig. 1(a), the line shapes of the XMCD curves in Fig. 2 are very nearly the same. This would suggest that the interface oxide layer does not contribute to the XMCD and must therefore have no net magnetic moment (as expected if either $\mathrm{CoO}$ or $\mathrm{Co}_{3} \mathrm{O}_{4}$ is formed, which are both room temperature paramagnets). The XMCD in this case is thus a measure of the effective polarization of Co atoms in the interface region, diluted by the presence of cobalt oxide. In order to quantify the Co spin-polarization as a function of oxidation time, we applied the magneto-optical sum rules to the XAS and XMCD intensities integrated over the absorption edges. ${ }^{15}$ This analysis yields the spin moment per hole, $\mu_{s} / n_{h}$, where $n_{h}$ is the total number of $3 d$ holes. In fact, $\mu_{s} / n_{h}$ corresponds to the net spin-polarization integrated over the unoccupied Co $3 d$ band $^{16}$ and can be extracted from the absorption spectra by following a standard procedure. ${ }^{12}$

As it was not possible in this experiment to reduce the $\mathrm{x}$-ray spot size below the width of the $\mathrm{NiFe} / \mathrm{Co}$ electrode, a varying background signal, caused by slight movements of the incident $\mathrm{x}$-ray beam with time, was also present in the XAS curves. The resulting error in $\mu_{s} / n_{h}$ was estimated by applying the sum rules to repeated measurements of the XAS and XMCD spectra obtained over several beam cycles. The values obtained (and their estimated error bars) are shown in Table I, from which it can be seen that the net Co $3 d$ spinpolarization $\left(\mu_{s} / n_{h}\right)$ initially increases with oxidation time (100 and $300 \mathrm{~s}$ ), reaching values consistent with previous measurements on thin Co layers. ${ }^{17}$ This is contrary to the expected decrease in the polarization caused by the formation of cobalt oxide at the interface. Further oxidation ( $600 \mathrm{~s}$ junction) leads to a reduction in $\mu_{s} / n_{h}$ compared to intermediate oxidation times, although this is less conclusive due to the significant error bars. 
TABLE I. Plasma oxidation times used for the samples studied, measured TMR ratios, and net Co $3 d$ spin-polarization, $\mu_{s} / n_{h}$, determined from the XAS and XMCD measurements. Errors bars are given in parentheses.

\begin{tabular}{cccc}
\hline \hline & \multicolumn{2}{c}{ TMR $(\%)$} & \\
\cline { 2 - 3 } Oxidation time (s) & Junction 1 & Junction 2 & $\mu_{S} / n_{h}\left(\mu_{B}\right)$ \\
\hline 0 & 0 & 0 & $0.60(2)$ \\
100 & $0.7(1)$ & $0.5(1)$ & $0.71(3)$ \\
300 & $13.6(1)$ & $11.4(1)$ & $0.74(6)$ \\
600 & $6.8(1)$ & $1.3(1)$ & $0.65(4)$ \\
\hline \hline
\end{tabular}

These results can be explained by considering the effective Co spin-polarization, measured by XMCD, to be the weighted average of the polarization, $P_{I}$, on the Co interface atoms bonded to the alumina layer, and the polarization, $P_{N}$, of near-interface Co atoms. According to the first-principles calculations discussed earlier, ${ }^{6}$ one would expect $P_{I}$ to increase with oxidation time as a greater portion of the interface is transformed from $\mathrm{Co}-\mathrm{Al}$ bonded to $\mathrm{Co}-\mathrm{O}$ bonded. However, over-oxidation of the interface will yield surplus $\mathrm{O}$ atoms that can form an oxide with the near-interface Co atoms, thereby reducing $P_{N}$. Once the interface is saturated with $\mathrm{Co}-\mathrm{O}$ bonds, continued barrier oxidation can only lead to a reduction in the effective Co spin-polarization $\left(\mu_{s} / n_{h}\right)$ as more of the near-interface $\mathrm{Co}$ atoms are incorporated into $\mathrm{CoO}$ or $\mathrm{Co}_{3} \mathrm{O}_{4}$.

The values of $\mu_{s} / n_{h}$ shown in Table I remain high for oxidation times up to $300 \mathrm{~s}$, suggesting that the interfaces in these MTJs are not saturated with $\mathrm{Co}-\mathrm{O}$ bonds at this point. This oxidation time also corresponds to the maximum in TMR obtained (Table I). When the oxidation time is increased to $600 \mathrm{~s}$, both the spin-polarization and TMR are reduced. At this point the reduction in $P_{N}$ caused by oxide formation must dominate both the effective Co spin polarization and the TSP. However, it should be noted that whereas XMCD probes the average polarization of the interface and near-interface atoms, spin-polarized tunneling will occur preferentially in regions where the barrier height and width are most favorable and is thus sensitive to barrier imperfections. This could account for the low TMR measured in the $100 \mathrm{~s}$ sample, despite the high value of $\mu_{s} / n_{h}$.

In summary, the electronic and magnetic structure of Co atoms near the Co/alumina interface in MTJs have been explored using XAS and XMCD, as a function of barrier oxidation time. It was found that the Co electronic structure tended to that of cobalt oxide as the barrier oxidation time increased. However, the effective Co spin polarization measured by XMCD was found to increase for moderate oxidation times, in contrast to the expected reduction caused by the formation of paramagnetic cobalt oxide. This reveals an increase in the spin polarization of the Co interface atoms when the bonding changes from $\mathrm{Co}-\mathrm{Al}$ to $\mathrm{Co}-\mathrm{O}$, that is sufficiently large to offset the dilution of the measured Co spin polarization due to cobalt oxide formation. Although we have not probed the spin polarization of $\mathrm{O}$ interface atoms in this experiment, our observations of a larger Co spin polarization as the number of $\mathrm{Co}-\mathrm{O}$ bonds increases is consistent with theoretical predictions ${ }^{6}$ for the bonding between exchange split Co $3 d$ and O $2 p$ orbitals, and may indeed reflect the formation of spin-polarized interface states in the barrier layer. Such states are thought to play a vital role in the mechanism for spin-polarized tunneling.

The authors are grateful for the support and assistance of DL staff and in particular Dr. Ian Kirkman during the beamtime.

${ }^{1}$ J. S. Moodera, L. R. Kinder, T. M. Wong, and R. Meservey, Phys. Rev. Lett. 74, 3273 (1995).

${ }^{2}$ E. Y. Tsymbal, O. N. Mryasov, and P. R. LeClair, J. Phys.: Condens. Matter 15, R109 (2003).

${ }^{3}$ R. Meservey and P. M. Tedrow, Phys. Rep. 238, 173 (1994).

${ }^{4}$ J. M. de Teresa, A. Barthélémy, A. Fert, J. P. Contour, F. Montaigne, and P. Seneor, Science 286, 507 (1999).

${ }^{5}$ J. M. MacLaren, X.-G. Zhang, W. H. Butler, and X. Wang, Phys. Rev. B 59, 5470 (1999).

${ }^{6}$ I. I. Oleinik, E. Yu. Tsymbal, and D. G. Pettifor, Phys. Rev. B 62, 3952 (2000).

${ }^{7}$ J. J. Sun, V. Soares, and P. P. Freitas, Appl. Phys. Lett. 74, 448 (1999).

${ }^{8}$ J. S. Moodera, E. F. Gallagher, K. Robinson, and J. Nowak, Appl. Phys. Lett. 70, 3050 (1997)

${ }^{9}$ L. Seve, W. Zhu, B. Sinkovic, J. W. Freeland, I. Coulthard, W. J. Antel, Jr., and S. S. P. Parkin, Europhys. Lett. 55, 439 (2001).

${ }^{10}$ J. W. Freeland, D. J. Keavney, R. Winarski, P. Ryan, J. M. Slaughter, R. W. Dave, and J. Janesky, Phys. Rev. B 67, 134411 (2003).

${ }^{11}$ N. D. Hughes and R. J. Hicken, J. Phys. D 35, 3153 (2002).

${ }^{12}$ C. T. Chen, Y. U. Idzerda, H. -J. Lin, N. V. Smith, G. Meigs, E. Chaban, G. H. Ho, E. Pellegrin, and F. Sette, Phys. Rev. Lett. 75, 152 (1995).

${ }^{13}$ T. J. Regan, H. Ohldag, C. Stamm, F. Nolting, J. Lüning, J. Stöhr, and R. L. White, Phys. Rev. B 64, 214422 (2001).

${ }^{14}$ B. T. Thole and G. van der Laan, Phys. Rev. B 38, 3158 (1988).

${ }^{15}$ B. T. Thole, P. Carra, F. Sette, and G. van der Laan, Phys. Rev. Lett. 68, 1943 (1992).

${ }^{16}$ G. van der Laan, J. Phys.: Condens. Matter 9, L259 (1997).

${ }^{17}$ Y. Huttel, G. van der Laan, T. K. Johal, N. D. Telling, and P. Bencok, Phys. Rev. B 68, 174405 (2003). 